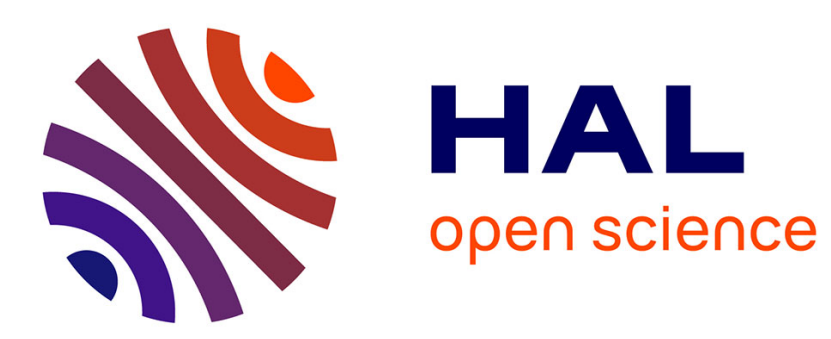

\title{
Overlap Regions and Grey Model-Based Approach for Interference Avoidance in Cognitive Radio Networks
}

Quach Minh Thao, Dramane Ouattara, Francine Krief, Hicham Khalifé, Mohamed Aymen Chalouf

\section{- To cite this version:}

Quach Minh Thao, Dramane Ouattara, Francine Krief, Hicham Khalifé, Mohamed Aymen Chalouf. Overlap Regions and Grey Model-Based Approach for Interference Avoidance in Cognitive Radio Networks. International Conference on Ubiquitous and Future Networks, IEEE ICUFN, 2013. hal00853905

\section{HAL Id: hal-00853905 https://hal.science/hal-00853905}

Submitted on 24 Aug 2013

HAL is a multi-disciplinary open access archive for the deposit and dissemination of scientific research documents, whether they are published or not. The documents may come from teaching and research institutions in France or abroad, or from public or private research centers.
L'archive ouverte pluridisciplinaire HAL, est destinée au dépôt et à la diffusion de documents scientifiques de niveau recherche, publiés ou non, émanant des établissements d'enseignement et de recherche français ou étrangers, des laboratoires publics ou privés. 


\title{
Overlap Regions and Grey Model-Based Approach for Interference Avoidance in Cognitive Radio Networks
}

\author{
Minh Thao Quach*, Dramane Ouattara*, Francine Krief*, Hicham Khalifé ${ }^{*}$ and Mohamed Aymen Chalouf ${ }^{\ddagger}$ \\ *Université Bordeaux 1, Bordeaux, France, Email: minh-thao.quach@labri.fr, dramane.ouattara@labri.fr, francine.krief@labri.fr \\ ${ }^{\dagger}$ Thales Communications \& Security, Colombes, France, Email: hicham.khalife@thalesgroup.com \\ ‡Université de Rennes 1, IUT de Lannion, France, Email: mohamed-aymen.chalouf@irisa.fr
}

\begin{abstract}
Ensuring high quality of communication while reducing interference remains a challenge in unplanned wireless mobile networks. The Cognitive Radio Ad Hoc Networks (CRAHNs) with cooperative sensing promise to be an appropriate solution for achieving high data rates. In this article, we evaluate the impact of cognitive radio transmissions on primary radio communications by characterizing precisely based on the position of the primary and secondary radio emitters, the vulnerable areas where interference causes impact to primary radios. Furthermore, we show that either reducing the overlap area or avoid the high density primary receivers area could considerably reduce the impact on the primary system while the secondary transmission is operating. Through OMNeT++ simulations, we illustrate that there is a dependency between the size of the overlap area: the smaller the overlap region, the smaller the impact on the receivers in this area. However, we also show that the distribution of the PR receivers are also a vital perspective on preventing interference. We hence investigate a solution to predict primary radio receivers position using the Grey Prediction Model.
\end{abstract}

\section{INTRODUCTION}

Cognitive Radio Ad Hoc Networks [1] have recently emerged as a new wireless technology that deals with the shortage of radio resources and the inefficiency in spectrum usage. In most common configurations, a Cognitive Radio user (CR) exploits the spectrum bands transparently to legacy users also called Primary Radio users (PR). Using its flexibility, cognitive radio identifies available frequency bands for opportunistically maintaining its transmissions.

However, this new technology also brings many challenges in spectrum management, protocol design and users mobility, due to the unstable nature of wireless links added to the hardly predictable behavior of PRs. Intermittent connectivity during an ongoing communication is one of the typical issues that cognitive radio protocols need to handle. Interruptions obviously happen as the transmission of a primary radio forces CRs to vacate on the selected band. On the other hand, when an overlap exists in the coverage area between the CR and PR transmitters, the undesired interference generated by the secondary transmitters on primary radio communications becomes difficult to control. For this reason, it is necessary to accurately characterize this overlap and its effect on every available channel for better spectrum selection.

In this paper, we present an exact computation of the vulnerable overlap area to characterize the impact of secondary transmission on a primary radio system. Our technique requires only the coordinate position and the coverage area (the reception zone) of CRs and PRs in order to calculate the region where these two zones geographically overlap. The size of the obtained area is directly linked to the interference on the existing PR receivers. It means that the smaller overlapping area is the less interference impact on on-going PR communications.

On the other hand, we also show the pratical case which the ratio of affected node is minor when the overlap encounters the low density of receivers, i.e. less receivers are placed and concerned when the intersection happens. We also propose a strategy to refine our computation of the overlap areas using the Grey Prediction Model. We can use this model to predict the sensitivity limit of each transmitter based on the Received Signal Strength Indication (RSSI), essential for achieving adaptive transmitting power for CRs and thereafter getting the nodes' position.

The remainder of this paper is structured as follows: in section II, we give an overview on the related work in the domain. We provide the accurate computation of the overlap area between PR and CR transmitters and the performance evaluation via OMNet++ in section III. In section IV, we introduce the Grey Model showing its capability to estimate the PR positions in the overlap region. Conclusion and future work are provided in section V.

\section{RELATED WORK}

Cognitive radio networks have recently attracted a large amount of research efforts from the community. Several papers have surveyed the already conducted work [1], [2] or have given guidelines for the future research directions [3]. The authors in [4], [5] argued that the performance in terms of throughput and delays of a secondary network could equal that of a standalone network by applying scaling laws when primary and secondary systems overlapped. A different overlapping perspective in Cognitive Radio Networks was proposed in [6], where the time overlap during sensing between the CRs was derived. The solution aimed at exploiting the waiting time for sensing while the other CRs report to a fusion center. 
Another work on the channel overlapping [7] discussed the partial frequency barrier overlapping when PR and $C R$ coexist over the same frequency. In fact, the authors focused on finding the minimum frequency separation between PR and $\mathrm{CR}$ to meet a provided certain average target bit error rate (BER). However, the positions of CR and PR are not clearly mentioned whether they coexisted geographically within an area or not. Both [6] and [7] addressed the overlapping aspects either in time or frequency that can be encountered in CRNs without a clear description of how PR receivers protection can be achieved.

PR receivers protection was studied in [8] in which a routing solution with consideration about overlapping was proposed. The routing mechanism ensures a perfect protection for PRs by selecting routes that avoid any overlap between primary radios and secondary radios coverage. However, the resources of the overlap region may be usable when the PR receivers are inactive or inexistent. The overlap region and its impact has been generally observed and evaluated in [9] but they have not noticed the case which low density of primary users was encountered within the overlap region. On the other hand, mobility prediction is also particularly notable in mobile networks.

Though we characterize the relationship between the overlap area and the effect on the primary system, the primary receivers density within this vulnerable area is vaguely known. We argue that the Grey Prediction Model which is able to estimate the mobile node position. The Grey Model theory [10] is known for the analysis of problems with incomplete or uncertain information. A proposal on the use of Grey Model in [11] defined a function that facilitates the detection of free-bands by the mobile cognitive radio equipment dedicated to the real-time patient's monitoring. This work basically proposed a predicting strategy which is based on machine learning techniques combined with the Grey Model system for performing a spectral prediction.

\section{THE OVERLAP REGION}

The location of these devices could be obtained by geolocation technologies [12] or location-based services as described in [13]. The location acquiring methods are out of the scope of this paper. We assume that the coverage area of $\mathrm{PR}$ and CR transmitters forms a perfect circle whose center is located at the position of the emitter. We denote by $O_{P}$ of coordinates $\left(x_{P}, y_{P}\right)$ and $O_{C}$ with $\left(x_{C}, y_{C}\right)$ the position of the PR and CR transmitters respectively, $d$ the distance between these two points, $R_{P}$ and $R_{C}$ the radius of their coverage disk respectively. Table I summarizes the used notations. Our goal is to calculate the overlap region and observe the impact of CR transmissions on the primary users within the area.

The obtained surface defines the region where PR receivers and CR potentially coexist. Noted that in some situations where no overlap region exists or when circles are inscribed (i.e. the PR reception area covers the CR disk or inversely), the overlap size can be easily estimated. Indeed, the overlap
TABLE I: Notations Table

\begin{tabular}{l|l} 
Symbols & Descriptions \\
\hline \hline$O_{P}\left(x_{P}, y_{P}\right)$ & $\begin{array}{l}\text { Center and corresponding co-ordination of a Primary } \\
\text { Radio (PR) transmitter }\end{array}$ \\
\hline$O_{C}\left(x_{C}, y_{C}\right)$ & $\begin{array}{l}\text { Center and corresponding co-ordination of a Cognitive } \\
\text { Radio (CR) node }\end{array}$ \\
\hline$R_{P}$ & Radius of the PR power transmission \\
\hline$R_{C}$ & Radius of the CR power transmission \\
\hline$d$ & $\begin{array}{l}\text { Distance between the center of a PR transmitter and a } \\
\text { CR }\end{array}$ \\
\hline$\theta_{P}$ & Angle of the sector created by the intersection of PR \\
\hline$\theta_{C}$ & Angle of the sector created by the intersection of CR \\
\hline$A\left(x_{A}, y_{A}\right)$ & $\begin{array}{l}\text { Intersection points } \\
\text { of CR and PR }\end{array}$ \\
\hline$G\left(x_{B}, y_{B}\right)$ & Inner point and on the boundary of the overlapped region \\
\hline$W\left(x_{G}, y_{G}\right)$ & Outer point and on the boundary of the overlapped region \\
\hline
\end{tabular}

area is obviously null in the former case and equals the size of the inscribed circle in the latter case.

\section{A. Overlap region computation}

Initially, in this computation we consider that the PR and CR positions are known, the radii are fixed and the PR transmitter is located at the origin of the coordinate system. The general case highlighted in Fig. 1, is obtained when the following condition is satisfied,

$$
d<R_{P}+R_{C} \text { and } d>R_{P} \text { and } d>R_{C} .
$$

Using classical geometry, we calculate the general overlap

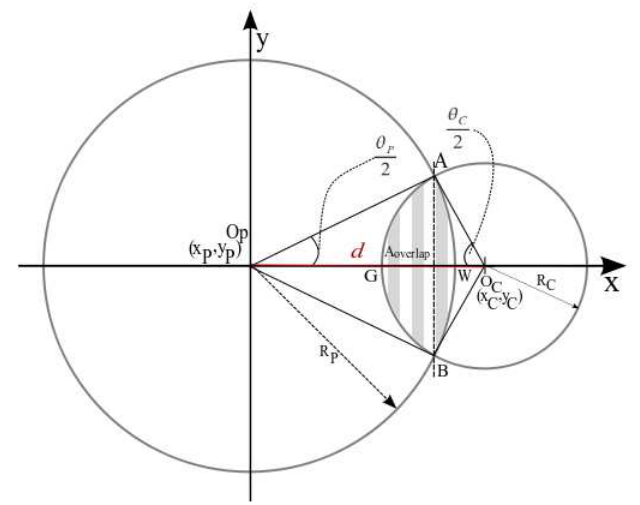

Fig. 1: General overlap case

region between PR and CR for this case in equation (2),

$$
\begin{aligned}
A_{\text {Overlap }} & =\frac{\theta_{C}}{2} R_{C}^{2}-R_{C}^{2}|\cos \beta| \cos \frac{\theta_{C}}{2} \\
& +\frac{\theta_{P}}{2} R_{P}^{2}-R_{C}|\cos \beta| R_{P} \cos \frac{\theta_{P}}{2}
\end{aligned}
$$

Where $\theta_{C}$ and $\theta_{P}$ are the angles formed at $O_{C}$ and $O_{P}$ with points $A$ and $B$ respectively, whereas $\beta$ is an intermediate variable for our computation given,

$$
\begin{aligned}
& \sin \beta=\frac{R_{P}^{2}-\left(R_{C}^{2}+x_{C}^{2}+y_{C}^{2}\right)}{2 * R_{C} * \sqrt{x_{C}^{2}+y_{C}^{2}}} ; \\
& \sin \frac{\theta_{C}}{2}=\frac{R_{C}}{R_{P}} *|\cos \beta| ; \\
& \sin \frac{\theta_{P}}{2}=|\cos \beta| .
\end{aligned}
$$


However, the case where $d<R_{C}<R_{P}$ shown in Fig. 2 is not included in equation (2). Therefore, to make our study complete, this particular situation is captured in equation (3).

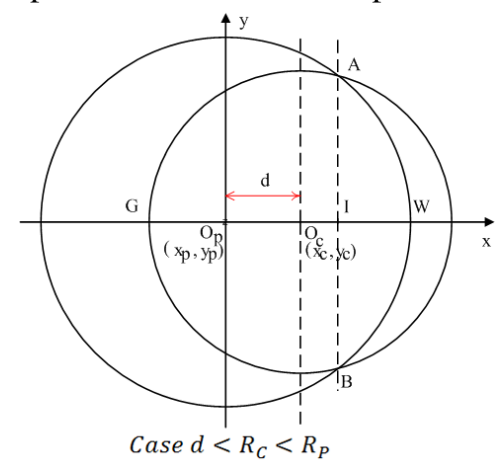

Fig. 2: Particular case overlap between $P R$ Transmitter and a CR Node

$$
\begin{aligned}
A_{\text {Overlap }} & =\frac{\theta_{P}}{2} R_{P}^{2}-R_{C} R_{P}|\cos \beta| \cos \frac{\theta_{P}}{2} \\
& +\Pi R_{C}^{2}-\frac{\theta_{C}}{2} R_{C}^{2} \\
& +R_{C}^{2}|\cos \beta| \cos \frac{\theta_{P}}{2} .
\end{aligned}
$$

The size of the overlap region depends on the reception zone of both the CR and the PR transmitters on a specific channel. Therefore, determining the size of the overlap is invital in characterizing the impact on the primary receivers. The smaller the region is, the less impact there is on the potential PR receivers operating on that channel.

As long as the PR receivers are envisaged and protected, the impact that the CRs transmissions have on PR communications can be contained. It is clear that in some specific cases it might be interesting to take also into account the PR deployment in the area. In fact, in some configurations, a small overlap region might contain a high number of PR receivers, in other words nodes may not be uniformly distributed in space. Through simulation we observe this phenomena in section III-C and we also discuss these situations in section IV.

\section{B. OMNet++ simulation descriptions}

The relationship between the overlap phenomena and its impact on the PR network is investigated using OMNeT++ simulator with the integrated MiXim framework. MiXim is a mixed framework that supports mobile and wireless simulations and offers detailed models of radio wave propagation, interference estimation, radio transceiver, power consumption and wireless MAC protocols as explained in [14].

The simulation was deployed on a playground size $3000 \mathrm{~m} \times 3000 \mathrm{~m}$, the PR transmitter is a typical $802.11 \mathrm{~b}$ single channel sender with the maximum transmission power of $110.11 \mathrm{~mW}$ that corresponds to a reception range of $475.8 \mathrm{~m}$. When the transmission is set down to $11.11 \mathrm{~mW}$, reception range hence decreases to about $103 \mathrm{~m}$. The number of receivers are kept varying from 10 to 200 nodes within the PR's transmitter reception region.
We developed a CR node on top of an adaptive 802.11 multi-channel implementation that allows nodes to switch between channels under specific conditions. Note here that the IEEE 802.11 was selected for simplicity reasons; however, our results can be generalized over any spectrum band. Cognitive radio transmission power was fixed at $55.11 \mathrm{~mW}$ and $5.5 \mathrm{~mW}$ that covers a distance of $377.77 \mathrm{~m}$ and $83.64 \mathrm{~m}$ respectively. This coverage distance is computed according to the maximum transmission power, wavelength, the path lost coefficient and a threshold for the minimal receiving power.

The built-in path loss propagation was used with default path loss coefficient alpha of 3.5. All the physical values were also kept by default as pre-defined in MiXim while the transmission power of the CR node was modified and set to be lower than the PRs transmission power. We placed the PR receivers in the disk area around the PR transmitter following various distributions. Every shown result is the outcome of 27 simulation runs while modifying the number of nodes and PR receivers distribution. Each run was performed in $500 \mathrm{~s}$ at least.

\section{Simulation results}

Fig. 3 and Fig. 4 show results for a scenario where over a single channel network we modify the overlap size and study its impact on PR receivers. We consider Uniform and Poisson distributions to deploy $\mathrm{PR}$ receivers around the primary radio emitter. The $\mathrm{x}$-axis illustrates the size of the overlap obtained from the location of the CR sender and calculated based on equations (2) or (3); the $y$-axis shows the ratio of impacted primary nodes. Lower coverage area in Fig. 5 Fig. 6 produces almost the similar observed results on the single channel experiments.

Apparently, the ratio of impacted PR receivers by the CRs transmissions grows linearly from $30 \%$ to more than $90 \%$ when the overlap size increases as observed in Fig. 3 and Fig. 4. However, our surprise comes from the observation that the ratio of affected PRs is almost independent of the number of PR receivers as different number of PR receivers are simulated. Decreasing the reception zones of these devices also gave us the same result as seen in Fig. 5 Fig. 6. This important result may seem intuitive with a uniform distribution where nodes are deployed inside the coverage disk with an equal probability, but we show here that with a different probability distribution (i.e. Poisson distribution), this tendency remains somehow similar.

Fig. 7 and Fig. 8 correspond to the second scenario where two channels are available with a PR transmitter observing different overlaps on each channel. In fact, the overlap on channel 1 is about $120000 \mathrm{~m}^{2}$ while on channel 2 the intersection between transmitter circles is around $500 \mathrm{~m}^{2}$. We study in the graphs depicted by Fig. 7 and Fig. 8, the distinctive ratio of affected nodes while modifying the number of PR receivers on both channels.

Again, the experiment is performed with various number of PR receivers within the PR transmitter's disks on both channels. The $\mathrm{x}$-axis shows the number PR receivers while 


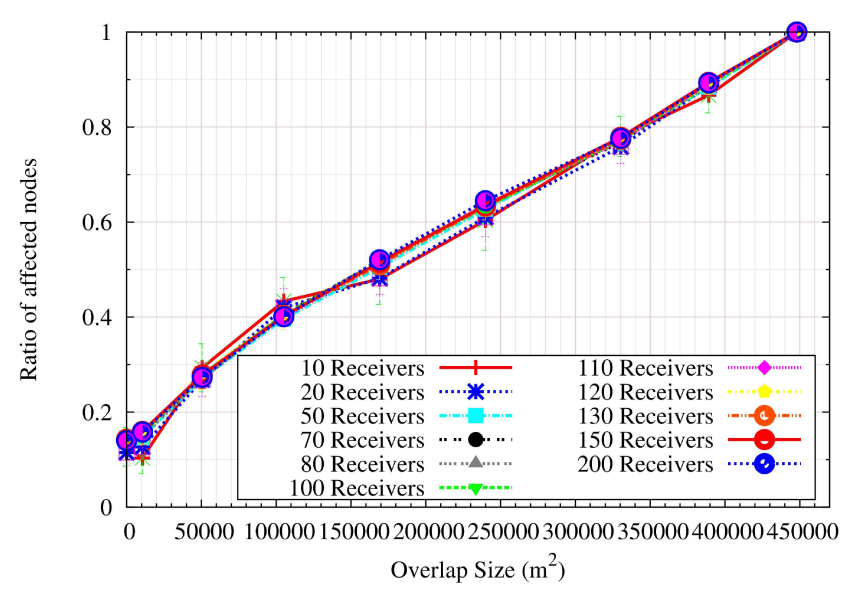

Fig. 3: Single Channel - Different Overlap region - Uniform Distribution

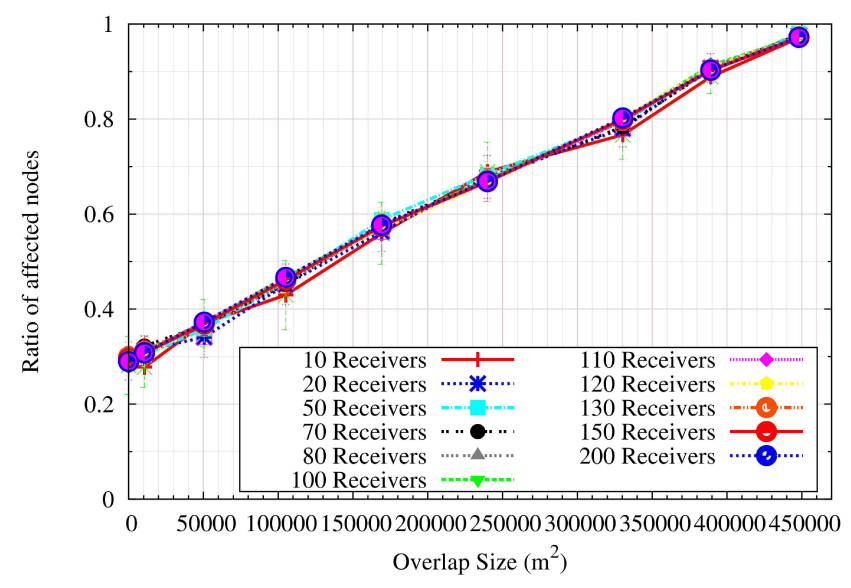

Fig. 4: Single Channel - Different Overlap region - Poisson Distribution

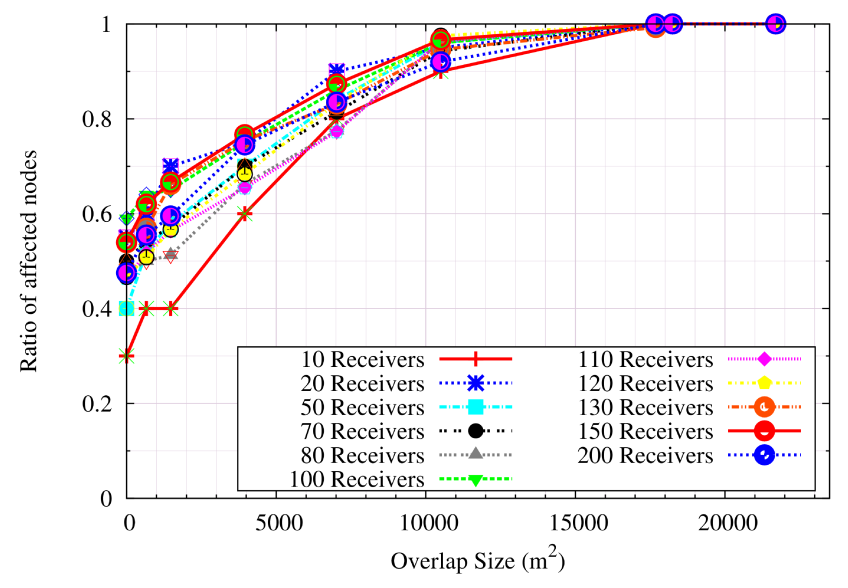

Fig. 5: Single Channel - Different Overlap region, smaller reception zone - Uniform Distribution

the $y$-axis illustrates the ratio of nodes being affected by the CR transmitters signal. Obviously, this ratio varies from $10 \%$ to almost $15 \%$ on channel 2 but from almost $34 \%$ to almost $40 \%$ on channel 1 when the receivers are distributed uniformly

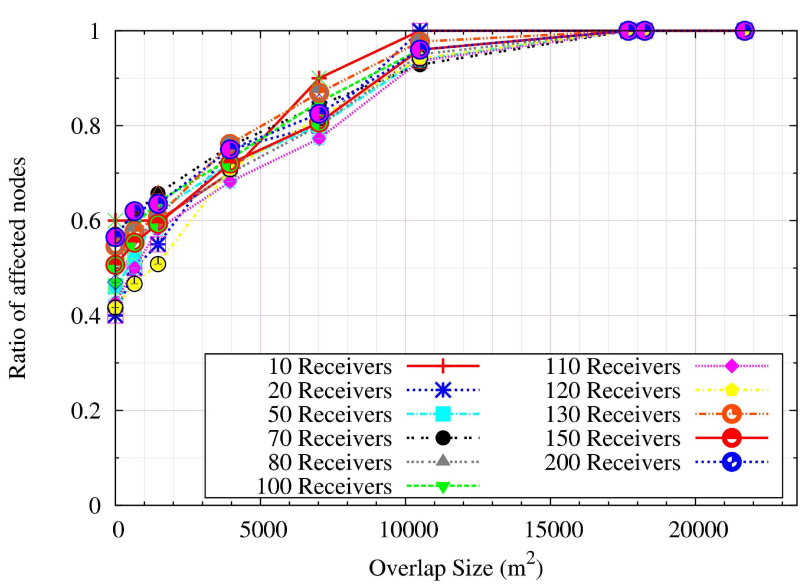

Fig. 6: Single Channel - Different Overlap region, smaller reception zone - Poisson Distribution

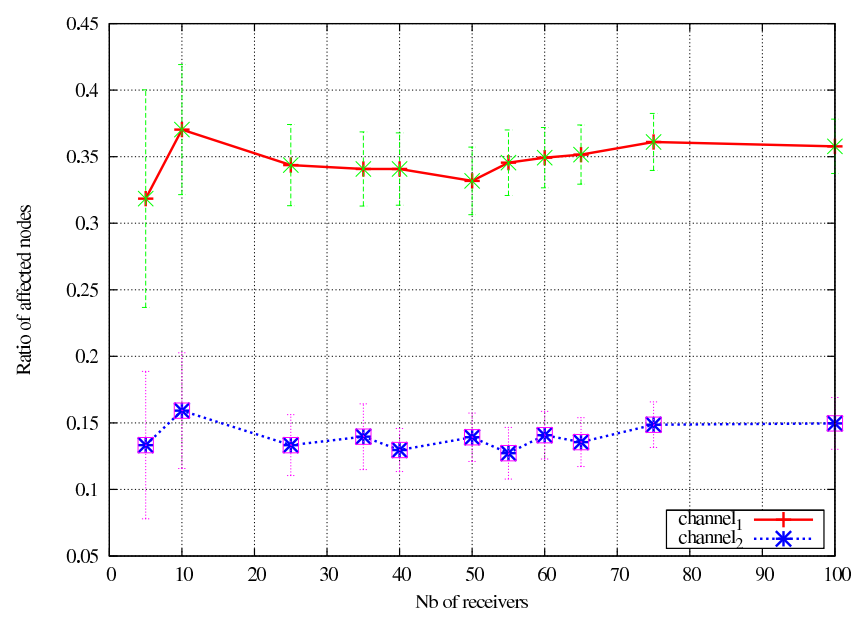

Fig. 7: Multi Channel - Different Overlap region - Uniform Distribution

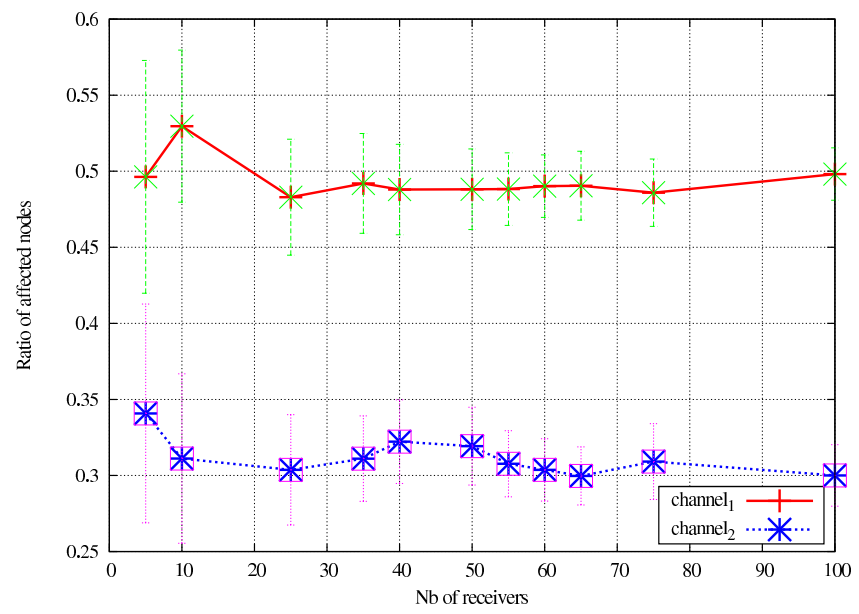

Fig. 8: Multi Channel - Different Overlap region - Poisson Distribution

within these disks (as in Fig. 7). These results corroborate the previous observation by showing again the limited impact of the number of PRs on the ratio of affected PRs for these distributions (i.e. Uniform and Poisson).

Though the relationship between overlap size and the impact 
is shown, we performed another experiments on single channel with different overlap size and arbitrarily deploy the receivers. As in, the primary users tends to be deployed further from the vulnerable area. For instance, even two hundred (200) receivers were deployed, the ratio of affected nodes were lower than the deployment of eighty (80) or fifty (50) receivers in Fig. 9 and Fig. 10 respectively were. Hence, envisaging the distribution over the overlap area and applying the proper prediction model to estimate the density of the receivers could be a new approach in protecting the primary receivers in general.

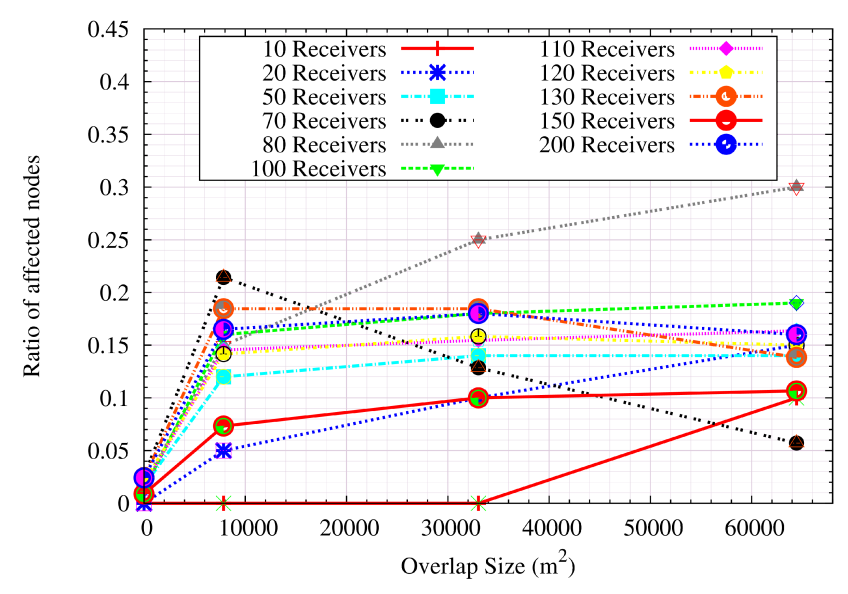

Fig. 9: Single Channel - Big Reception Zone - Arbitrary Deployment

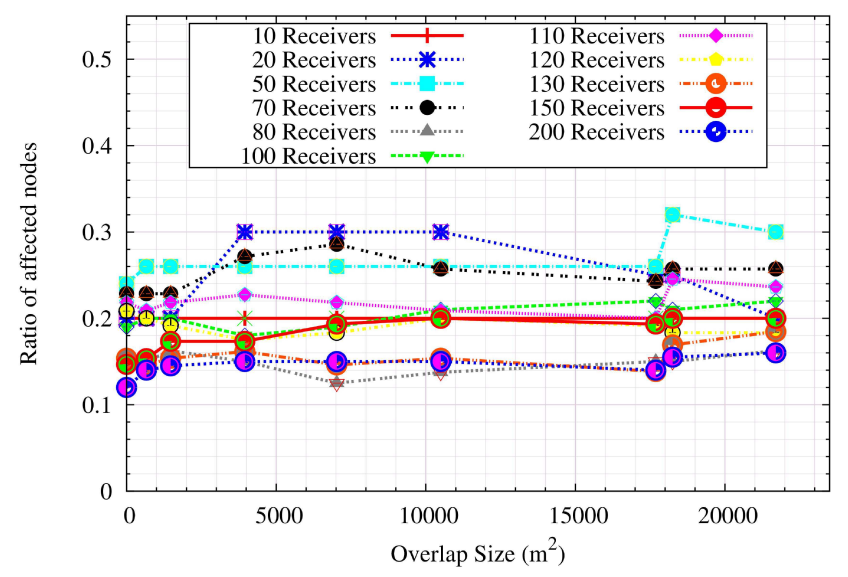

Fig. 10: Single Channel - Small Reception Zone - Arbitrary Deployment

In summary, the simulation results reflect the relationship between the overlap area and the impact on the primary receptions with homogeneous distributions of the $\mathrm{PR}$ receivers. However, positions of PRs may be impacted by practical considerations such as obstacles buildings, mountains, etc. that prevent regions of the coverage area from containing receivers.

Therefore, a prediction model to estimate the location of these devices is required. The Grey prediction model appears to be an appropriate tool to solve the problem. With Grey model, based on the previous receiver power, we can predict the mobility of the mobile node at time $t+t^{\prime}$; hence, we can predict the location where a node may exist.

\section{The GREy PREDiction Model}

We assume that unlicensed users or the secondary radio users cooperatively sense the spectrum and share the sensing results with the others. Based on exchanged informations and received signal power, $\mathrm{CR}$ is able to estimate the mobile PR receiver position. Considering the uncertainty generated by this context (mobile) and the path loss, it is more reliable to model the mapping function while taking into account these uncertainties. An example of modeling the environment with the Grey Model (GM) technique for mobility (distance $d$ ) estimation/prediction is given.

1) The problem modeling with the $G M(1,1)$ : One of the main advantages of the Grey Model resides in its statistical ability to settle for a minimum input data to achieve its predictions. The $\operatorname{GM}(1,1)$ is the most used in prediction systems [15].

- The system takes as input a sequence of values, the received signal that characterizes the received power from each PR transmitter in the overlap region; with $X^{0}=\left[x^{0}(1), x^{0}(2), x^{0}(3), \ldots, x^{0}(n)\right]$, the initial distribution corresponding to each PR power received at time $[1, . ., n]$ from the overlap region and $x^{0}(d)$ their distance $d$ according to their transmit/received power.

- From the initial distribution, a new sequence $X^{1}$ is generated by using Accumulating Generation Operation (AGO) $X^{1}=\left[x^{1}(1), x^{1}(2), x^{1}(3), \ldots, x^{1}(n)\right]$

- The first order differential equation obtained from $X^{1}$ is given by: $\frac{d x^{1}(t)}{d t}+a x^{1}(t)=b, a$ is the coefficient that reflects the trend and $b$ is the predictive control coefficient expressing the portion of the information; known and unknown part of the information model.

- The parameter estimation or coefficients of the matrix $[a, b]^{T}$ can be obtained by the method of least squares knowing that $[a, b]^{T}=\left(B^{T} B\right)^{-1} B^{T} y_{N}$, where

$$
B=\left(\begin{array}{cc}
-\frac{1}{2}\left(x^{1}(1)+x^{1}(2)\right. & 1 \\
-\frac{1}{2}\left(x^{1}(2)+x^{1}(3)\right. & 1 \\
-\frac{1}{2}\left(x^{1}(3)+x^{1}(4)\right. & 1 \\
\vdots & \vdots \\
-\frac{1}{2}\left(x^{1}(n-1)+x^{1}(n)\right. & 1
\end{array}\right)
$$

and $y_{N}=\left[x^{0}(2), x^{0}(3), x^{0}(4), \ldots, x^{0}(n)\right]^{T} ; n$ being the size of the sequence.

- The distance $(t=d)$ estimation function becomes: $\hat{x}^{1}(t)=\left(x^{1}(1)-\frac{b}{a}\right) e^{-a t}+\frac{b}{a}$

- The distance variation according to the received power and hence the future position of PR receiver could be obtained by: $\hat{x}^{0}(d+1)=\hat{x}^{1}(d+1)-\hat{x}^{1}(d)$

\section{A. Simulation related to the static transmitter $(C R)$ and mobile receivers $(P R)$}

The relationship between transmission power and reception power in free space can be approximated by [16]: 


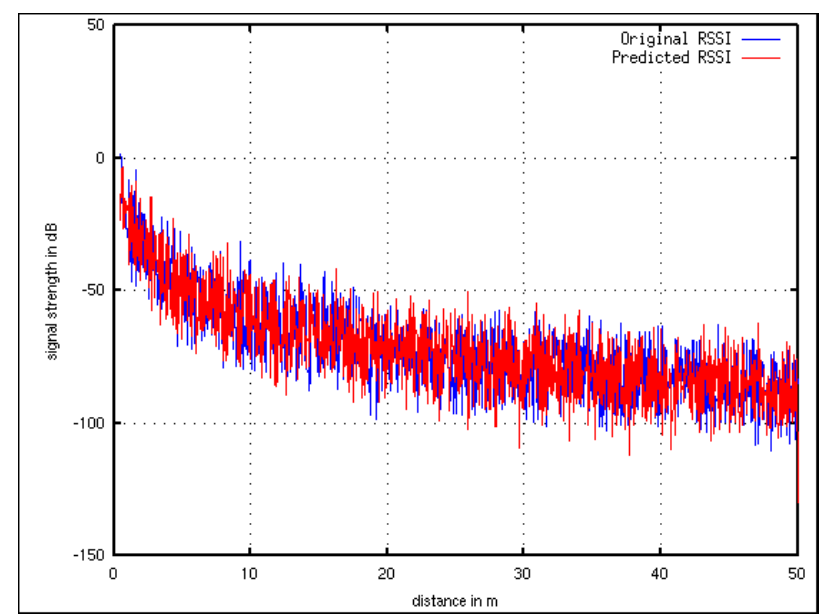

Fig. 11: Received power and PR position prediction

$$
P_{r}(d)=\frac{P_{t} G_{t} G_{r} \lambda^{2}}{(4 \pi d)^{2} L}
$$

Where $P_{r}$ and $P_{t}$ are received and transmitted power respectively, $G_{r}$ and $G_{t}$ denote the antenna gains of receiver and transmitter, $L$ the system loss factors (a.k.a. filter losses and antenna losses), $d$ the distance between the transmitter and the receiver and $\lambda$ is the wavelength of the transmit signal in meters. Thus, knowing the PR positions based on their transmitting/received power by the $\mathrm{CR}$ node, the system (i.e. Cognitive Radio emitter) could estimate the changes in their position and number from the overlap region. According to this information, CR node could adapt its transmit parameters (power) for interferences avoidance.

Fig. 11 shows the relationship between the received signal and the distance. The future position is predicted based on this received signal and the current position. This example (Fig. 11) allows us to estimate the different positions of the PRs in overlap region and to characterize the overlap region with PRs mobility. The sensitivity range for this case has a limit which approaches the $-100 \mathrm{dBm}$. These results can also be the basis for adaptive transmission power selection.

\section{CONCLUSION}

We have computed the size of the overlap area and evaluated the ratio of primary receivers that potentially experienced intereferece within this area. Our mathematical formula can be used as a routing metric or in other layers mechanisms to push forward the coexistence of primary and secondary users in cognitive radio environments. With the simulation results on Omnet++, we clarified the relationship between the overlap region and the impact on the primary receivers on an ongoing transmission. Furthermore, the results also explained the situation where the overlap region was useable when it encountered the low density area of the primary users.

At the same time, we have proposed a strategy to predict the PR receivers or mobile nodes position within the overlap area based on Grey Model. These results provide a basis for reasonable and autonomous choice of transmission parameters and channel selection to minimize interferences in cognitive radio networks. We can also explore the possibility of using Grey prediction model to estimate positions then optimize transmission power of secondary emitters accordingly. Clearly, such mechanism can be exploited to reduce power consumption thus pushing towards the green communication paradigm.

\section{ACKNOWLEDGMENT}

This work is supported by LICoRNe project, funded in part by the National Agency for Research in France - ANR (Agence Nationale de la Recherche).

\section{REFERENCES}

[1] I. F. Alyikdiz, Won-Yeol, and K. R. Chowdhury, "CRAHNs: Cognitive Radio Adhoc Networks," Ad Hoc Networks Journal (Elsevier), 2009.

[2] I. F. Akyildiz, W.-Y. Lee, M. C. Vuran, and S. Mohanty, "NeXt generation/dymanic spectrum access/cognitive radio wireless newtork: A survey," in Computer Communications, 2006, pp. 2127-2152.

[3] H. Khalife, N. Malouch, and S. Fdida, "Multihop cognitive radio networks: to route or not to route," Network, IEEE, vol. 23, no. 4, pp. $20-25$, july-august 2009.

[4] C. Li and H. Dai, "On the Throughput Scaling of Cognitive Radio Adhoc Networks," in IEEE INFOCOM 2011, 2011.

[5] W. Huang and X. Wang, "Throughput and Delay Scaling of General Cognitive Networks," in IEEE INFOCOM 2011, 2011.

[6] H. N. Vu and H. Y. Kong, "Overlap cooperative spectrum sensing with energy comparing in cognitive radio networks," in Strategic Technology (IFOST), 2010 International Forum on 13-15 Octorber 2010, 2010.

[7] E. Bedeer, M. Marey, O. Dobre, and K. Baddour, "On partially overlapping coexistence for dynamic spetrum access in cognitive radio," in 2011 IEEE 16th Workshop on Computer Aided Modeling and Design of Communication Links and Network (CAMAD), 2011.

[8] K. R. Chowdhury and I. F. Akyildiz, "CRP: A Routing Protocol for Cognitive Radio Ad Hoc Networks," IEEE JSAC, (Journal of Selected Areas in Communications), Special Issue on Cognitive Radio Networking and Communications, vol. 29, no. 4, April 2011.

[9] M. T. Quach and H. Khalife, "The impact of overlap regions in cognitive radio networks," in Wireless Days (WD), 2012 IFIP, Nov., pp. 1-3.

[10] D. Julog, "The basis of grey theory," Huazhong University of Science and Technology Press, 2002.

[11] D. Ouattara, F. Krief, M. A. Chalouf, and O. Ahmdi, "Spectrum sensing improvement in cognitive radio networks for real-time patients monitoring," in Mobihealth, Paris, France, 2012.

[12] K. F. King, "Geolocation and federalism on the internet: Cutting internet gambling's gordian knot," The Columbia Science and Technology Law Review, 2009.

[13] S. Wang, J. Min, and B. K. Yi, "Location based services for mobiles: Technologies and standards," in IEEE international conference on communication (ICC), vol. 19, 2008.

[14] K. Wessel, M. Swigulski, A. Köpke, and D. Willkomm, "Mixim the physical layer an architecture overview," in OMNeT++ 2009: Proceedings of the 2nd International Workshop on OMNeT++ (hosted by SIMUTools 2009). ICST, Brussels, Belgium, Belgium: ICST (Institute for Computer Sciences, Social-Informatics and Telecommunications Engineering), 2009.

[15] C. S., Y. Lin, Z. G., Z. C., D. S., and D. Chao, "Short-term wind power prediction based on combined grey-markov model," pp. 17051711, 2011.

[16] D. N. Ekram Hossain and Z. Han, "Dynamic spectrum access and management, cognitive radio networks," Cambridge University Press, 2009. 